\title{
Exponential Cluster Synchronization of Neural Networks with Proportional Delays
}

\author{
Nian Feng, Ye Wu, Weiping Wang, Lin Zhang, and Jinghua Xiao \\ School of Science, Beijing University of Posts and Telecommunications, Beijing 100876, China \\ Correspondence should be addressed to Weiping Wang; shiya666888@126.com
}

Received 15 January 2015; Revised 9 March 2015; Accepted 24 March 2015

Academic Editor: Kun Liu

Copyright (C) 2015 Nian Feng et al. This is an open access article distributed under the Creative Commons Attribution License, which permits unrestricted use, distribution, and reproduction in any medium, provided the original work is properly cited.

\begin{abstract}
Exponential cluster synchronization of neural networks with proportional delays is studied in this paper. Unlike previous constant delay or bounded time delay, we consider the time-varying proportional delay is unbounded, less conservative, and more widely applied. Furthermore, we designed a novel adaptive controller based on Lyapunov function and inequality technique to achieve exponential cluster synchronization for neural networks and by using a unique way of equivalent system we proved the main conclusions. Finally, an example is given to illustrate the effectiveness of our proposed method.
\end{abstract}

\section{Introduction}

In the past most researches focus on the complete synchronization of neural networks, which means that all the nodes of the whole network reach the same synchronization state, and this has achieved significant progress [1-6]. However, in many real world networks, it is to complete several functions at the same time, which implies that the network reaches several different states at last, which is a different synchronization type: cluster synchronization. As a particular synchronization phenomenon, cluster synchronization is achieved when the dynamical nodes realize complete synchronization in each subgroup which is called cluster, but no synchronization appears among the different clusters. Owing to the significant application in biological science and communication engineering, many results have been available for cluster synchronization of complex dynamical networks [7-10]. Ma et al. [9] proposed a coupling scheme with cooperative and competitive weight-couplings to realize cluster synchronization for the connected chaotic networks. In [10], cluster synchronization of complex networks was investigated with centralized adaptive pinning control.

In fact, there exist natural time delays in the operation of systems which are due to the limited communication speed when information transmission exists, extra time required by the sensor to get the measurement information, computation time required for generating the control inputs, and the execution time required for the inputs being acted, and it may cause undesirable dynamic behaviors such as oscillation and instability. Recently, it has been revealed that some types of delayed neural networks (DNNs) exhibit some complicated dynamics and even chaotic behaviors if the parameters and time delays are appropriately chosen. Therefore, dynamic behaviors, especially the cluster synchronization problems of DNNs, have been extensively considered. So far, most studied models of synchronization problem are neural networks (NNs) with constant delays, time-varying and bounded delays, distributed delays, and so on [11-18]. In [17], the authors discuss the synchronization of complex dynamical networks by only designing the adaptive controllers, while we introduce adaptive strategies not only to the coupling strengths but also to the feedback gains. Dahms et al. [18] investigate the stability of synchronized states in delay-coupled networks where synchronization takes place in groups of different local dynamics or in cluster states in networks with identical local dynamics. In addition, delaydependent stability criteria and synchronization control laws are related to the size of delay so that they can be used to design some better networks according to the allowed time delays of networks. So the cluster synchronization of the neural network with time delay is the right direction for further research.

The proportional delay is one of many delay types and is objectively existent. Unlike previous constant delay or 
bounded time delay, the time-varying proportional delay is unbounded, less conservative, and more widely applied. For example, in Web quality of service (QoS) routing decision, the proportional delay is usually required [19-23]. The proportional delay system as an important mathematical model often rises in some fields such as physics, biology systems, and control theory and it has attracted many scholars' interest [24, 25]. If the QoS routing algorithms based on neural networks with proportional delays are proposed, they will be the most suitable algorithms corresponding to the actual situation. Since a neural network usually has a spatial nature due to the presence of an amount of parallel pathways of a variety of axon sizes and lengths, it is reasonable that the proportional delays are introduced in the neural networks according to the topology structure and parameters of neural networks. The proportional delay function $\tau(t)=(1-q) t(0<q<1)$ is a monotonically increasing function about the time $t$, which will be convenient to control the network's running time according to the time delays of network. So far a few results of dynamic behaviors of DNNs with proportional delays have been reported in [26-31]. Zhou [26, 30,31] has discussed the global exponential stability, asymptotic stability of cellular neural networks (CNNs), and the research of synchronization with proportional delays, by employing matrix theory and constructing Lyapunov function. Dissipativity of a class of CNNs with proportional delays has been investigated by using the inner product properties in [27]. So far, to the best of the author's knowledge, there are few studies with the cluster synchronization of the neural network with proportional delays.

Motivated by the above discussions, we creatively take the element of the proportional delays into the model of neural networks to achieve the exponential cluster synchronization. As an intersection of multibranches of learning, this model will be closer to the reality and much more pertinence for concrete questions. Whether in the study of delays or in the research of cluster synchronizations, the paper has important referring value. The main novelty of our contribution is as follows.

(1) The cluster synchronization model with proportional delay is considered.

(2) The coupling strength of each edge in the system only depends on its local information.

(3) We use a unique way of equivalent system to prove the main results.

This paper is organized as follows. In Section 2, the models and preliminaries are presented. In Section 3, by the application of Lyapunov function, some inequality analysis technique, and the way of equivalent system, the results can be obtained, which is about the cluster synchronization of the delayed neural network. In Section 4, a numerical example and its simulation are given to illustrate the effectiveness of the proposed method. Conclusions are presented in Section 5 .

\section{Model Description and Preliminaries}

Consider a delayed neural network (DNN) with proportional delay of $N$ coupled identical nodes, with each node being an $n$-dimensional dynamical system, which can be described by the following equations [26]:

$$
\begin{aligned}
& \dot{x}_{i}(t)=-C x_{i}(t)+A f\left(x_{i}(t)\right)+B f\left(x_{i}\left(q_{i} t\right)\right) \\
&+\sum_{j=1, j \neq i}^{N} g_{i j}(t) \Gamma\left(x_{j}(t)-x_{i}(t)\right), \quad t \geq 1, \\
& x_{i}(s)=x_{i 0}, \quad q \leq s \leq 1, i=1,2,3, \ldots, N,
\end{aligned}
$$

where $N \geq 2$ denotes the number of nodes (neurons) in the network; $x_{i}(t)=\left[x_{i 1}, x_{i 2}, x_{i 3}, \ldots, x_{i n}\right]^{T} \in \mathbb{R}^{n}$ is the state variable of node $i ; C=\operatorname{diag}\left(c_{1}, c_{2}, c_{3}, \ldots, c_{n}\right) \in \mathbb{R}^{n \times n}$ is a diagonal matrix with positive entries. $A=\left(a_{i j}\right)_{n \times n}$ and $B=\left(b_{i j}\right)_{n \times n}$ are the corresponding weight and delayed weight matrices; $f\left(x_{i}(t)\right)=\left(f\left(x_{i 1}, x_{i 2}, x_{i 3}, \ldots, x_{i n}\right)\right)^{T} \in \mathbb{R}^{n}$ denotes a nonlinear activation function of nodes; $G=\left(g_{i j}\right)_{n \times n}$ is the coupling matrix between nodes. If there is a connection between node $i$ and node $j$ at time $t$, then $g_{i j}(t)=g_{j i}(t)>$ $0(i \neq j)$; otherwise $g_{i j}(t)=g_{j i}(t)=0(i \neq j)$. The diagonal item of the matrix $G$ is $g_{i i}(t)=-\sum_{j=1, j \neq i}^{N} g_{i j}(t)$. In this paper, the network is connected in the sense that there are no isolated clusters in the network, which means that the matrix $G$ is symmetric and irreducible [10]. $q_{i}(i=1,2,3, \ldots, N)$ are proportional delay coefficient and satisfy $0<q_{i} \leq 1$, $q=\min _{1 \leq i, j \leq N}\left\{q_{i}\right\}$, and $q_{i} t=t-\left(1-q_{i}\right) t$ in which $\left(1-q_{i}\right) t$ corresponds to the time delay, and $\left(1-q_{i}\right) t \rightarrow \infty$, as $q \neq 1$, $t \rightarrow \infty$. Furthermore, the neural networks described in (1) possess initial conditions of $x_{i}(s)=x_{i 0}, s \in[q, 1], x_{i 0}(i=$ $1,2,3, \ldots, N)$ are constants, and $x_{i 0}=\left[x_{i 01}, x_{i 02}, \ldots, x_{i 0 n}\right]^{T} . \Gamma$ is inner matrix, and $\Gamma>0$.

According to the characteristics of the matrix $G$, system network (1) is easily turned to the following further simplified equations:

$$
\begin{aligned}
\dot{x}_{i}(t)= & -C x_{i}(t)+A f\left(x_{i}(t)\right)+B f\left(x_{i}\left(q_{i} t\right)\right) \\
& +\sum_{j=1}^{N} g_{i j}(t) \Gamma x_{j}(t), \quad t \geq 1, \\
x_{i}(s)= & x_{i 0}, \quad q \leq s \leq 1, \quad i=1,2,3, \ldots, N .
\end{aligned}
$$

Assume the whole $N$ nodes are divided into $M$ clusters. Without loss of generality, the sets of subscripts of these clusters are $C_{1}=\left\{1,2,3, \ldots, N_{1}\right\}, C_{2}=\left\{N_{1}+1, N_{1}+\right.$ $\left.2, \ldots, N_{1}+N_{2}\right\}, \ldots, C_{M}=\left\{N_{1}+\cdots+N_{m-1}+1, \ldots, N\right\}$, where $N_{1}+N_{2}+\cdots+N_{M}=N$. The network is considered to realize 
cluster synchronization, when the states of nodes achieve the following states [32]:

$$
\begin{gathered}
x_{1}(t)=x_{2}(t)=\cdots=x_{N_{1}}(t)=s_{i}(t), \quad i=1,2, \ldots, N_{1}, \\
x_{n_{1}+1}(t)=x_{N_{1}+2}(t)=\cdots=x_{N_{1+N_{2}}}(t)=s_{i}(t) \\
i=N_{1}+1, N_{1}+2, \ldots, N_{1}+N_{2}, \\
\vdots \\
X_{N_{1}+\cdots+N_{m-1}+1}(t) \\
=x_{N_{1}+\cdots+N_{m-1}+2}(t)=\cdots=x_{N}(t)=s_{i}(t) \\
i=N_{1}+1, N_{1}+2, \ldots, N_{1}+N_{2},
\end{gathered}
$$

where $s_{i}(t)=\left(s_{i 1}(t), s_{i 2}(t), \ldots, s_{i n}(t)\right)^{T}$ is the desired state of node $i$ at time $t$ and satisfies

$$
\dot{s}_{i}(t)=-C s_{i}(t)+A f\left(s_{i}(t)\right)+B f\left(s_{i}\left(q_{i} t\right)\right) .
$$

In order to make the network (1) realize cluster synchronization, an input control is added in (1) which is designed as follows:

$$
u_{i}(t)=-\sum_{j=1, j \neq i}^{N} g_{i j}(t) \Gamma\left(x_{j}(t)-x_{i}(t)\right) .
$$

According to the characteristics of the matrix $G$, the input (5) is easily turned to the following further simplified equation:

$$
u_{i}(t)=-\sum_{j=1}^{N} g_{i j}(t) \Gamma x_{j}(t)
$$

where the coupling strength $g_{i j}(t)=\left(g_{i j}^{1}, g_{i j}^{2}, \ldots, g_{i j}^{n}\right)^{T}$ between nodes adopts the following local adaptive strategy:

$$
\begin{gathered}
\dot{g}_{i j}(t)=\beta \|\left[x_{j}(t)-s_{j}(t)\right]-\left[x_{i}(t)-s_{i}(t)\right] \\
+\left[x_{j}\left(q_{j} t\right)-s_{j}\left(q_{j} t\right)\right] \\
-\left[x_{i}\left(q_{i} t\right)-s_{i}\left(q_{i} t\right)\right] \|,
\end{gathered}
$$

where $\beta>0$ is the adaptive gain.

Before starting to discuss the cluster synchronization, we make the following assumption.

Assumption 1. The activation function $f\left(x_{i}(t)\right)$ satisfies that $f: \mathbb{R}^{n} \rightarrow \mathbb{R}^{n},|f(u)-f(v)| \leq L|u-v|$, where $u, v \in \mathbb{R}^{n}$ and $L$ is positive constant.

Let us consider transformation defined by [27]

$$
y_{i}(t)=x_{i}\left(e^{t}\right), \quad i=1,2, \ldots, N .
$$

Case 1. When $e^{t} \geq 1$, then $t \geq 0$ and $\dot{y}_{i}(t)=\dot{x}_{i}\left(e^{t}\right) e^{t}$; that is,

$$
\dot{x}_{i}\left(e^{t}\right)=\dot{y}_{i}(t) e^{-t} .
$$

Let $e^{t}=z, z \geq 1$; then (8) is written as

$$
\dot{x}_{i}(z)=\dot{y}_{i}(t) z^{-1} \text {. }
$$

According to the above various formulas one gets

$$
\begin{aligned}
\dot{y}_{i}(t) z^{-1}= & -C x_{i}(z)+A f\left(x_{i}(z)\right)+B f\left(x_{i}\left(q_{i} z\right)\right) \\
& +\sum_{j=1}^{N} g_{i j}(z) \Gamma x_{j}(z)+u_{i}(t) .
\end{aligned}
$$

That is to say,

$$
\begin{aligned}
\dot{x}_{i}\left(e^{t}\right)= & -C x_{i}\left(e^{t}\right)+A f\left(x_{i}\left(e^{t}\right)\right)+B f\left(x_{i}\left(q_{i} e^{t}\right)\right) \\
& +\sum_{j=1}^{N} g_{i j}\left(e^{t}\right) \Gamma x_{j}\left(e^{t}\right)+u_{i}\left(e^{t}\right) .
\end{aligned}
$$

According to (8), we have

$x_{i}\left(q_{i} e^{t}\right)=x_{i}\left(e^{t+\ln q_{i}}\right)=y_{i}(t+\ln q)=y_{i}\left(t-\tau_{i}\right)$.

Using (8) and (13) in (12) one gets

$$
\begin{array}{r}
\dot{y}_{i}(t)=e^{t}\left\{-C y_{i}(t)+A f\left(y_{i}(t)\right)+B f\left(y_{i}\left(t-\tau_{i}\right)\right)\right. \\
\left.+\sum_{j=1}^{N} G_{i j}(t) \Gamma u_{j}(t)\right\}+U_{i}(t), \quad t \geq 0 .
\end{array}
$$

Case 2. When $e^{t} \in[q, 1]$, we have

$$
x_{i}\left(e^{t}\right)=x_{i 0}, \quad t \in[-\tau, 0],
$$

where $\tau=\max _{1 \leq i \leq N}\left(\tau_{i}\right), \tau_{i}=-\ln q_{i}$. Thus, the initial function associated with system (12) is given by

$$
Y_{i}(s)=x_{i 0}, \quad-\tau \leq s \leq 0, i=1,2, \ldots, N
$$

The analysis is completed.

Remark 2. Conversely, let $\tau_{i}=-\ln q_{i}, \tau=\max _{1 \leq i \leq N}\left(\tau_{i}\right)$; by (8), (16) and (14) can be turned to (1), which means that the transformation is reversible and equivalent.

Above all, let

$$
\begin{array}{r}
y_{i}(t)=x_{i}\left(e^{t}\right), \\
w_{i}(t)=s_{i}\left(e^{t}\right), \\
G_{i j}(t)=g_{i j}\left(e^{t}\right), \\
U_{i}(t)=u_{i}\left(e^{t}\right), \\
i=1,2,3, \ldots, N .
\end{array}
$$


System (1) with the input control (5) can be equivalently turned to the following neural network with a fixed delay, which can be described as

$$
\begin{gathered}
\dot{y}_{i}(t)=e^{t}\left\{\begin{array}{c}
-C y_{i}(t)+A f\left(y_{i}(t)\right)+B f\left(y_{i}\left(t-\tau_{i}\right)\right) \\
\left.+\sum_{j=1}^{N} G_{i j}(t) \Gamma y_{j}(t)\right\}+U_{i}(t),
\end{array}\right. \\
\begin{array}{c}
\dot{w}_{i}(t)=e^{t}\left\{-C w_{i}(t)+A f\left(w_{i}(t)\right)+B f\left(w_{i}\left(t-\tau_{i}\right)\right)\right\}, \\
U_{i}(t)=e^{t}\left\{\begin{array}{c}
\left.-\sum_{j=1, j \neq i}^{N} G_{i j}(t) \Gamma\left(y_{j}(t)-y_{i}(t)\right)\right\}, \\
\dot{G}_{i j}(t)=e^{t}\left\{\beta \|\left[y_{j}(t)-w_{j}(t)\right]-\left[y_{i}(t)-w_{i}(t)\right]\right.
\end{array}\right. \\
+\left[y_{j}\left(t-\tau_{i}\right)-w_{j}\left(t-\tau_{i}\right)\right] \\
\left.-\left[y_{i}\left(q_{i} t\right)-w_{i}\left(q_{i} t\right)\right] \|\right\}, \\
s \in[-\tau, 0] ; i=1,2, \ldots, N,
\end{array}
\end{gathered}
$$

where $t \geq 0, \tau=\max _{1 \leq i \leq N}\left(\tau_{i}\right), \tau_{i}=-\ln q_{i}>0$, and $x_{i}(s)=$ $x_{i 0} \in C\left([-\tau, 0], \mathbb{R}^{n}\right), i=1,2, \ldots, N$, is constant continuous function.

Remark 3. The translations are to convert the desired cluster synchronization state to another equivalent control system, which is convenient to study the cluster synchronization. Namely, the state when the original system realizes the cluster synchronization means that the control system and the original system realized synchronization.

Definition 4. System (18) and (19) is said to be exponentially synchronized if there exist constants $M \geq 1$ and $\lambda>0$ such that

$$
\begin{array}{r}
\sum_{i=1}^{n}\left|y_{i}(t)-w_{i}(t)\right| \leq M \sum_{i=1}^{n} \sup _{\tau \leq s \leq 0}\left|y_{i}(s)-w_{i}(s)\right| e^{-\lambda t}, \\
t \geq 0,
\end{array}
$$

where the constant $\lambda$ is defined as the exponential synchronization rate.

The goal of this paper is to prove that system (1) with adaptive control (5) can achieve the desired cluster synchronization. Now it is equivalently turned to prove that system (18) and (19) can meet the exponential synchronization. In order to prove that it satisfies the exponential synchronization, the following dynamic equations of synchronization error can be obtained:

$$
\begin{gathered}
\dot{h}_{i}(t)=e^{t}\left\{-C h_{i}(t)+A F\left(h_{i}(t)\right)+B F\left(h_{i}\left(t-\tau_{i}\right)\right)\right. \\
\left.+\sum_{j=1}^{N} G_{i j}(t) \Gamma y_{j}(t)\right\}+U_{i}(t)
\end{gathered}
$$

$$
\begin{aligned}
& =e^{t}\left\{-C h_{i}(t)+A F\left(h_{i}(t)\right)\right. \\
& \left.\quad+B F\left(h_{i}\left(t-\tau_{i}\right)\right)+\sum_{j=1}^{N} G_{i j}(t) \Gamma h_{j}(t)\right\},
\end{aligned}
$$

where $t \geq 0, i=1,2, \ldots, N$, and $h_{i}(t)=y_{i}(t)-w_{i} ; F\left(h_{i}(t)\right)=$ $f\left(y_{i}(t)\right)-f\left(w_{i}(t)\right) ; F\left(h_{i}\left(t-\tau_{i}\right)\right)=f\left(y_{i}\left(t-\tau_{i}\right)\right)-f\left(w_{i}\left(t-\tau_{i}\right)\right)$, $\tau_{i}=-\ln q_{i}>0$.

Thus the goal of this paper is further equivalently turned into the proof of the dynamic equations of synchronization error to achieve an exponential stabilization.

\section{Main Results}

Theorem 5. Assume that Assumption 1 holds. If $|A| L+$ $|B| L e^{\sigma \tau}+\sigma-1<|C|$ is true, system (18) and (19) can achieve exponential stabilization with the synchronization rate $\alpha=$ $\sigma-1>0$, where $L>0 ; \sigma>1 ; \tau=\max _{1 \leq i \leq N}\left(\tau_{i}\right)$.

Proof. According to the definition of $\dot{h}_{i}(t)$ and Assumption 1, we get

$$
\begin{gathered}
\left|F\left(h_{i}(t)\right)\right| \leq L\left|h_{i}(t)\right|, \\
\left|F\left(h_{i}\left(t-\tau_{i}\right)\right)\right| \leq L\left|h_{i}\left(t-\tau_{i}\right)\right| .
\end{gathered}
$$

Consider the following function:

$$
H_{i}(t)=e^{\sigma t}\left|h_{i}(t)\right|, \quad \sigma>1
$$

From (24) to (26) one gets

$$
\begin{aligned}
& \dot{H}_{i}(t)=\sigma e^{\sigma}\left|h_{i}(t)\right|+e^{\sigma t} \frac{d\left|h_{i}(t)\right|}{d t} \\
& =\sigma e^{\sigma}\left|h_{i}(t)\right|+e^{\sigma t} \frac{h_{i}(t) \dot{h}_{i}(t)}{\left|h_{i}(t)\right|} \\
& =\sigma e^{\sigma}\left|h_{i}(t)\right| \\
& +e^{\sigma t}\left(\left(h_{i}(t) * e^{t}\right.\right. \\
& *\left[-C h_{i}(t)+A F\left(h_{i}(t)\right)\right. \\
& +B F\left(h_{i}\left(t-\tau_{i}\right)\right) \\
& \left.\left.+\sum_{j=1}^{N} G_{i j}(t) \Gamma h_{j}(t)\right]\right) \\
& \left.\cdot\left|h_{i}(t)\right|^{-1}\right)
\end{aligned}
$$




$$
\begin{aligned}
& \leq \sigma e^{\sigma}\left|h_{i}(t)\right| \\
& +e^{\sigma t} e^{t}\left\{\begin{array}{l}
-|C|\left|h_{i}(t)\right| \\
+
\end{array}\right. \\
& +\sum_{j=1}^{N}|A| L\left|h_{i j}(t)\right|+|B| L\left|h_{i}\left(t-\tau_{i}\right)\right| \\
& =\sigma H_{i}(t)+e^{t}\left\{\begin{array}{c}
-|C| H_{i}(t)+|A| L H_{i}(t) \\
+|B| L e^{\sigma \tau_{i}} H_{i}\left(t-\tau_{i}\right)
\end{array}\right. \\
& \left.+\sum_{j=1}^{N}\left|G_{i j}(t)\right| \Gamma H_{i}(t)\right\} .
\end{aligned}
$$

Now construct the following positive Lyapunov functional as follows:

$$
\begin{aligned}
V(t)= & e^{-t} \sum_{i=1}^{N} H_{i}(t)+|B| \sum_{i=1}^{N} L e^{\sigma \tau_{i}} \int_{t-\tau_{i}}^{t} H_{i}(s) d s \\
& +\frac{1}{2 \beta} \sum_{\varepsilon}\left(h_{i j}-G_{i j}(t)\right)^{T}\left(h_{i j}-G_{i j}(t)\right),
\end{aligned}
$$

where $t \geq 0, \sigma>1 ; \varepsilon$ is the edges' set of the network; $h_{i j}$ is a constant.

Then the differential of $V(t)$ is

$$
\begin{aligned}
\dot{V}(t)= & -e^{-t} \sum_{i=1}^{N} H_{i}(t)+e^{-t} \sum_{i=1}^{N} \dot{H}_{i}(t) \\
& +|B| \sum_{i=1}^{N} L e^{\sigma \tau_{i}}\left(H_{i}(t)-H_{i}\left(t-\tau_{i}\right)\right) \varepsilon \varepsilon \\
& -\frac{1}{\beta} \sum_{\varepsilon}\left(h_{i j}-G_{i j}(t)\right)^{T} \dot{G}_{i j}(t) .
\end{aligned}
$$

According to (27), we can get

$$
\begin{aligned}
& \dot{V}(t)=-e^{-t} \sum_{i=1}^{N} H_{i}(t) \\
& +e^{-t} \sum_{i=1}^{N}\left\{\sigma H_{i}(t)+e^{t}\left\{-|C| H_{i}(t)\right.\right. \\
& +|A| L H_{i}(t) \\
& +|B| L e^{\sigma \tau_{i}} H_{i}\left(t-\tau_{i}\right) \\
& \left.\left.+\sum_{j=1}^{N}\left|G_{i j}(t)\right| \Gamma H_{i}(t)\right\}\right\}
\end{aligned}
$$

$$
\begin{aligned}
& +|B| \sum_{i=1}^{N} L e^{\sigma \tau_{i}}\left(H_{i}(t)-H_{i}\left(t-\tau_{i}\right)\right) \\
& +\sum_{\varepsilon}\left(h_{i j}-G_{i j}(t)\right)^{T} e^{t} \beta
\end{aligned}
$$$$
\cdot \|\left[y_{j}(t)-w_{j}(t)\right]-\left[y_{i}(t)-w_{i}(t)\right]
$$$$
+\left[y_{j}\left(q_{j} t\right)-w_{j}\left(q_{j} t\right)\right]
$$$$
-\left[y_{i}\left(q_{i} t\right)-w_{i}\left(q_{i} t\right)\right] \|
$$$$
=\sum_{i=1}^{N}\left\{(\sigma-1) e^{-t}-|C|+|A| L\right.
$$$$
\left.+|B| L e^{\sigma \tau_{i}}+\sum_{j=1}^{N} G_{i j}(t) \Gamma\right\} H_{i}(t)
$$$$
-\sum_{\varepsilon}\left(h_{i j}-G_{i j}(t)\right)^{T}
$$

$$
\begin{aligned}
\cdot e^{t}\{\beta & \|\left[y_{j}(t)-w_{j}(t)\right]-\left[y_{i}(t)-w_{i}(t)\right] \\
+ & {\left[y_{j}\left(q_{j} t\right)-w_{j}\left(q_{j} t\right)\right] } \\
& \left.-\left[y_{i}\left(q_{i} t\right)-w_{i}\left(q_{i} t\right)\right] \|\right\}
\end{aligned}
$$$$
=\sum_{i=1}^{N} e^{\sigma t}\left\{(\sigma-1) e^{-t}-|C|+|A| L+|B| L e^{\sigma \tau_{i}}\right.
$$$$
\left.+\sum_{j=1}^{N}\left|G_{i j}(t)\right| \Gamma\right\}\left|h_{i}(t)\right|
$$$$
-\sum_{\varepsilon}\left(h_{i j}-G_{i j}(t)\right)^{T} e^{t}
$$

$$
\begin{gathered}
\cdot\left\{\beta \|\left[y_{j}(t)-w_{j}(t)\right]-\left[y_{i}(t)-w_{i}(t)\right]\right. \\
+\left[y_{j}\left(q_{j} t\right)-w_{j}\left(q_{j} t\right)\right] \\
\left.-\left[y_{i}\left(q_{i} t\right)-w_{i}\left(q_{i} t\right)\right]\right\} .
\end{gathered}
$$

According to Assumption 1 and (25), we can get

$$
\begin{aligned}
\dot{V}(t) \leq & \sum_{i=1}^{N} e^{\sigma t}\left\{(\sigma-1)-|C|+|A| L+|B| L e^{\sigma \tau}\right\}|| h_{i}(t) \mid \\
& +\sum_{i=1}^{N} \sum_{j=1}^{N} e^{\sigma t}\left|G_{i j}(t)\right| \Gamma\left|h_{i}(t)\right| \\
& +\sum_{\varepsilon}\left(h_{i j}-G_{i j}(t)\right)^{T} \\
& \cdot e^{t} \beta \|\left[y_{j}(t)-w_{j}(t)\right]-\left[y_{i}(t)-w_{i}(t)\right]
\end{aligned}
$$




$$
\begin{gathered}
+\left[y_{j}\left(q_{j} t\right)-w_{j}\left(q_{j} t\right)\right] \\
-\left[y_{i}\left(q_{i} t\right)-w_{i}\left(q_{i} t\right)\right] \| \\
\leq \sum_{i=1}^{N} e^{\sigma t}\left\{(\sigma-1)-|C|+|A| L+|B| L e^{\sigma \tau}\right\}|| h_{i}(t) \mid \\
+\sum_{\varepsilon}\left(h_{i j}-G_{i j}(t)\right)^{T} \\
\cdot e^{t} \beta \|\left[y_{j}(t)-w_{j}(t)\right] \\
-\left[y_{i}(t)-w_{i}(t)\right]+\left[y_{j}\left(q_{j} t\right)-w_{j}\left(q_{j} t\right)\right] \\
-\left[y_{i}\left(q_{i} t\right)-w_{i}\left(q_{i} t\right)\right] \| .
\end{gathered}
$$

By the conditions of the theorems $|A| L+|B| L e^{\sigma \tau}+\sigma-$ $1<|C|$, and, for any edge $(i, j) \in \varepsilon$, there exists the value $h_{i j}$ which is bigger than or equal to the corresponding edge strength $G_{i j}(t)$, and we get

$$
\dot{V}(t) \leq 0, \quad t \geq 0,
$$

which means $V(t) \leq V(0)$, for $t \geq 0$. Then, by (25) and (28), one has

$$
\sum_{i=1}^{N} e^{\sigma t} e^{-t}\left|h_{i}(t)\right| \leq V(t) \leq V(0)
$$

It follows from (28) that

$$
\begin{aligned}
V(0) & =\sum_{i=1}^{N}\left|h_{i}(0)\right|+|B| \sum_{i=1}^{N} L e^{\sigma \tau_{i}} \int_{-\tau_{i}}^{0} H_{i}(s) d s \\
& \leq \sum_{i=1}^{N}\left|h_{i}(0)\right|+|B| \sum_{i=1}^{N} L \tau_{i} e^{\tau_{i}} \sup _{-\tau_{i} \leq s \leq 0} H_{i}(s) \\
& \leq \sum_{i=1}^{N}\left|h_{i}(0)\right|+|B| \sum_{i=1}^{N} L \tau e^{\tau} \sup _{-\tau \leq s \leq 0} H_{i}(s) \\
& \leq \max _{1 \leq i \leq N}\left(1+|B| L \tau e^{\tau}\right) \sum_{i=1}^{N} \sup _{-\tau \leq s \leq 0} H_{i}(s),
\end{aligned}
$$

where $M=\max _{1 \leq i \leq N}\left(1+|B| L \tau e^{\tau}\right) \geq 1$.

All told, one gets

$$
\sum_{i=1}^{n}\left|y_{i}(t)-w_{i}(t)\right| \leq M \sum_{i=1}^{n} \sup _{-\tau \leq s \leq 0}\left|y_{i}(s)-w_{i}(s)\right| e^{-\alpha t},
$$

where $\alpha=\sigma-1, \sigma>1$.

The proof is completed.

Remark 6. System (18) and (19) is asymptotically synchronized if the following conditions are satisfied: (1) the exponential synchronization rate is zero; that is, $\lambda=0$ and $M>1$; then $\sum_{i=1}^{n}\left|y_{i}(t)-w_{i}(t)\right| \leq M \sum_{i=1}^{n} \sup _{-\tau \leq s \leq 0}\left|y_{i}(s)-w_{i}(s)\right|$, $t \geq 0$; (2) $\lim _{t \rightarrow \infty}\|h(t)\|=0$.
According to the foregoing contents $y_{i}(t)=x_{i}\left(e^{t}\right), w_{i}(t)=$ $s_{i}\left(e^{t}\right)$, one gets

$$
\begin{array}{r}
\sum_{i=1}^{n}\left|x_{i}\left(e^{t}\right)-s_{i}\left(e^{t}\right)\right| \leq M \sum_{i=1}^{n} \sup _{-\tau \leq s \leq 0}\left|x_{i}\left(e^{s}\right)-s_{i}\left(e^{s}\right)\right| e^{-\alpha t}, \\
e^{t} \geq 1 .
\end{array}
$$

Let $e^{t}=\eta$; then $\eta \geq 1$ and $t=\ln \eta \geq 0$; let $e^{s}=\xi$; then $s=\ln \xi \in[-\tau, 0]$ and $\xi \in[q, 1]$. Thus, it follows from (36) that

$$
\sum_{i=1}^{N}\left|x_{i}(\eta)-s_{i}(\eta)\right| \leq M \sum_{i=1}^{n} \sup _{q \leq \xi \leq 1}\left|x_{i}(\xi)-s_{i}(\xi)\right| e^{-\alpha \ln \eta},
$$

$\eta \geq 1$.

Let $\eta=t$; the following inequality is obtained:

$$
\sum_{i=1}^{N}\left|x_{i}(t)-s_{i}(t)\right| \leq M \sum_{i=1}^{n} \sup _{q \leq \xi \leq 1}\left|x_{i 0}-s_{i 0}\right| e^{-\alpha \ln t}
$$

$t \geq 1$.

Thus, control system (4) and original system (1) are considered to be exponentially synchronized, and the exponential synchronization rate is less than $\alpha$ because of $0<$ $\ln t<t$; that is, $\ln t / t<1$, as $t \geq 1$.

Thus, the following theorem is derived.

Inference 1. Assume that Assumption 1 holds. If $|A| L+$ $|B| L e^{\sigma \tau}+\sigma-1<|C|$ is true, system (1) with the input control (5) can achieve an exponential cluster synchronization for the desired state, with the exponential rate being $\alpha=\sigma-1>0$, where $L>0 ; \sigma>1 ; \tau=\max _{1 \leq i \leq N}\left(\tau_{i}\right),-\ln q_{i}>0$.

The proof is omitted.

\section{Illustrative Examples}

In the following simulation, the whole neural network is divided into three clusters, which means that $M=3$. The desired state $s_{i}(t)$ of node $i$ at time $t$ is chosen as the average states of all the nodes in the same cluster at time $t$ as follows:

$$
\begin{aligned}
s_{1}(t) & =s_{2}(t)=\cdots=s_{N_{1}}(t) \\
& =\frac{x_{1}(t)+x_{2}(t)+\cdots+x_{N_{1}}(t)}{N_{1}}, \\
s_{N_{1}+1}(t) & =s_{N_{1}+2}(t)=\cdots=s_{N_{1}+N_{2}}(t) \\
& =\frac{x_{N_{1}+1}(t)+x_{N_{1}+2}(t)+\cdots+x_{N_{1}+N_{2}}(t)}{N_{1}},
\end{aligned}
$$




$$
\begin{aligned}
& s_{N_{1}+N_{2}+1}(t) \\
& =s_{N_{1}+N_{2}+2}(t)=\cdots=s_{N_{1}+N_{2}+N_{3}}(t) \\
& =\frac{x_{N_{1}+N_{2}+1}(t)+x_{N_{1}+N_{2}+2}(t)+\cdots+x_{N_{1}+N_{2}+N_{3}}(t)}{N_{1}},
\end{aligned}
$$

where the three clusters are $N_{1}, N_{2}$, and $N_{3}$, respectively.

Consider the following delayed neural networks (DCNNs):

$$
\dot{y}_{i}(t)=-C y_{i}(t)+A f\left(y_{i}(t)\right)+B f\left(y_{i}\left(t-\tau_{i}\right)\right),
$$

where $y(t)=\left[y_{1}(t), y_{2}(t)\right]^{T}, f(y)=\left[\tanh \left(y_{1}\right), \tanh \left(y_{2}\right)\right]^{T}$, and the parameters are

$$
\begin{gathered}
C=\left[\begin{array}{ll}
1 & 0 \\
0 & 1
\end{array}\right], \quad A=\left[\begin{array}{cc}
2.0 & -0.1 \\
-5.0 & 3.0
\end{array}\right], \\
B=\left[\begin{array}{rr}
-1.5 & -0.1 \\
-0.2 & -2.5
\end{array}\right] .
\end{gathered}
$$

The system meets Assumption 1 and Theorem 5 with $L=1$. The chaotic behavior of system (40) with the initial condition $\left[y_{1}(s), y_{2}(s)\right]^{T}=[0.4,0.6]^{T},(-1 \leq s \leq 0)$, has already been reported in the case of $\tau_{i}=1 s$ [33] (see Figure 1).

Example 1. Referring to the classical model, consider the following improved model with $N$ nodes:

$$
\begin{aligned}
\dot{y}_{i}(t)= & -C y_{i}(t)+A f\left(y_{i}(t)\right)+B f\left(y_{i}\left(t-\tau_{i}\right)\right) \\
& +\sum_{j=1}^{N} g_{i j}(t) \Gamma\left(x_{j}(t)-s_{j}(t)\right) .
\end{aligned}
$$

The system meets Assumption 1 and Theorem 5 with $L=$ $1, \beta=0.2$,

$$
\begin{aligned}
& C=\left[\begin{array}{ll}
2 & 0 \\
0 & 2
\end{array}\right], \quad A=\left[\begin{array}{cc}
2.0 & -0.1 \\
-5.0 & 3.0
\end{array}\right], \\
& B=\left[\begin{array}{rr}
-1.5 & -0.1 \\
-0.2 & -2.5
\end{array}\right], \quad \Gamma=\left[\begin{array}{ll}
1 & 0 \\
0 & 1
\end{array}\right],
\end{aligned}
$$

$y(t)=\left[y_{1}(t), y_{2}(t)\right]^{T}$, and $f(y)=\left[\tanh \left(y_{1}\right), \tanh \left(y_{2}\right)\right]^{T}$, where $q_{i}=0.5(i=1,2, \ldots, N)$ is constants, which is the proportional delay coefficient.

In the simulation experiment, a BA scale-free neural network is needed, which is constructed with $m=m_{0}=5$ and the neural network size $N=50$. The construction method of the BA scale-free neural network can refer to [34]. Suppose the whole network will reach three clusters with the cluster size being 14,8 , and 28 , respectively. The initial states $y_{i 1}(t)$ and $y_{i 2}(t), i=1,2, \ldots, 50$, are randomly distributed between $[-1,1]$ and $[-5,5]$.

As shown in Figure 2(a), for $t>2 \mathrm{~s}, 14$ black curves changed into one black curve, 8 red curves changed into

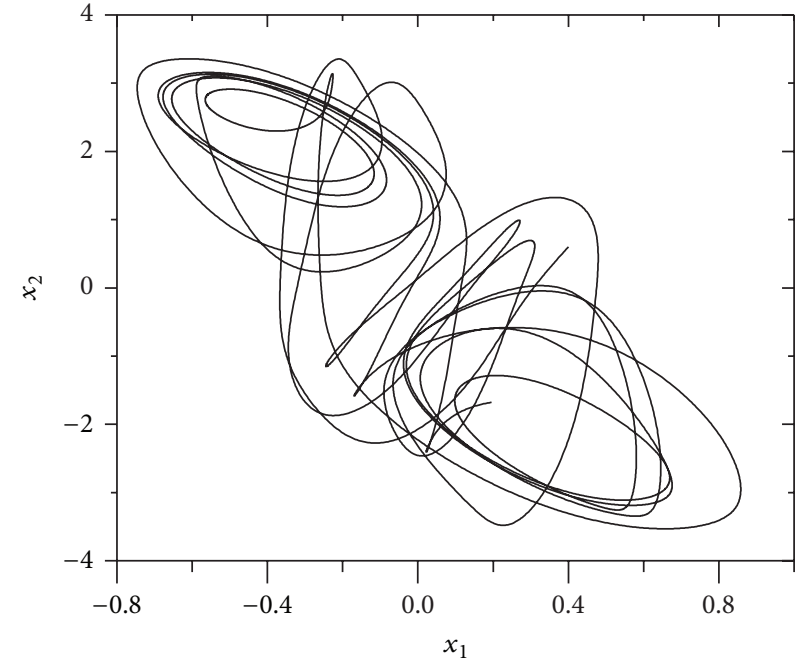

FIGURE 1: The chaotic DCNNs with $\tau_{i}=1 s$ in (40).

one red curve, and the remaining 28 blue curves changed into one blue curve; that is, the 50 state lines of $x_{i 1}(t),(i=$ $1,2, \ldots, 50)$, in system gradually merged into 3 lines, which means that each node of the neural network realizes the desired cluster synchronization. It reflects that the number of the nodes in the cluster influences the rate of the cluster synchronization. Then in Figure 2(b), for $t>1.8 \mathrm{~s}$, it can still be observed that 14 black curves changed into one black curve, 8 red curves changed into one red curve, and the remaining 28 blue curves changed into one blue curve; that is, the 50 state lines of $x_{i 1}(t),(i=1,2, \ldots, 50)$, in system gradually merged into 3 lines, which means that each node of the neural network realizes the desired cluster synchronization. It reflects that the number of the nodes in the cluster influences the rate of the cluster synchronization. So the conclusion is that the larger the cluster in the delay systems, the slower the rate of the cluster synchronization with the same way of the coupling mode.

Figure 3 depicts the synchronization error of the state variables between system (42) and the desired state, which makes it easy to see that the exponential convergence rate of the red curves within all the curves of the 3 cluster's nodes is relatively quick (14 were black, 8 red, and 24 blue classification). And it proves that from another aspect the number of nodes affects the rate of cluster synchronization. Furthermore the shape of the curves indicates that system (42) realizes the cluster synchronization with the exponential synchronization rules, and it is feasible to further study the cluster synchronization of the neural system with proportional delay through the exponential analysis of the synchronization.

In Figure 4, it can be seen that when $t>2 \mathrm{~s}$, the edge coupling strength $g_{i j}(t)$ between node $i$ and node $j$ is kept constant in all its dimensions. Combined with Figure 2, it is found that when the neural network realizes cluster synchronization, the edge coupling strengths are fixed, which implies that the left side of (7) will be zero. According to Figure 4, it is shown that most edge coupling strengths are 


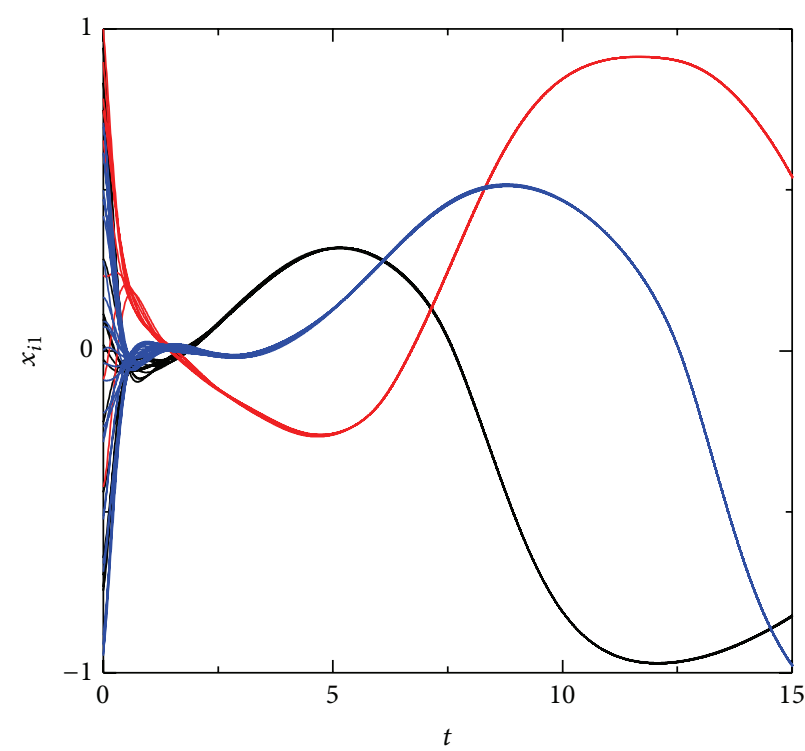

(a)

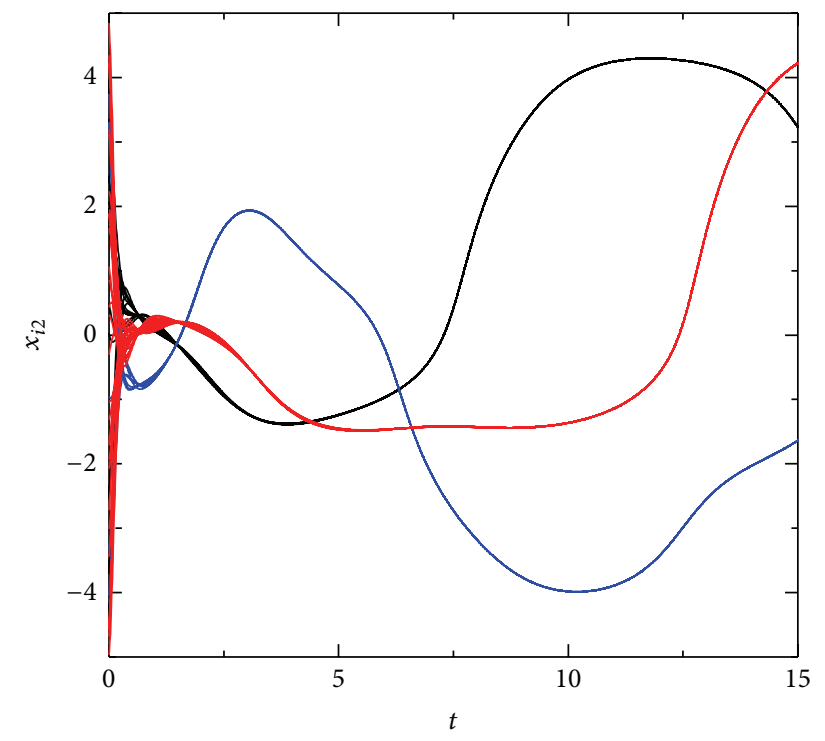

(b)

FIGURE 2: Cluster synchronization in the BA scale-free neural network with the proportional delay and the adaptive gain $\beta=0.2$ and the proportional delay coefficient $q_{i}=0.5$. (a) The curve of the state of $x_{i 1}$ changing over time; (b) the curve of the state of $x_{i 2}$ changing over time.



FIGURE 3: The synchronization error between system (42) and the system which the desired state is equivalently turned into.

less than 0.20 , and only a small amount of edge coupling strengths is distributed in the interval $[0.2,0.255]$. If we do not use the adaptive coupling strength in the neural network with the proportional delay, the network will realize the cluster synchronization only when all the edge coupling strengths are greater than 0.20 , which means that a lot of unnecessary coupling strengths are wasted. All in all, it is easy to design coupling intensity for each edge by using the adaptive coupling strength, in order to achieve the desired

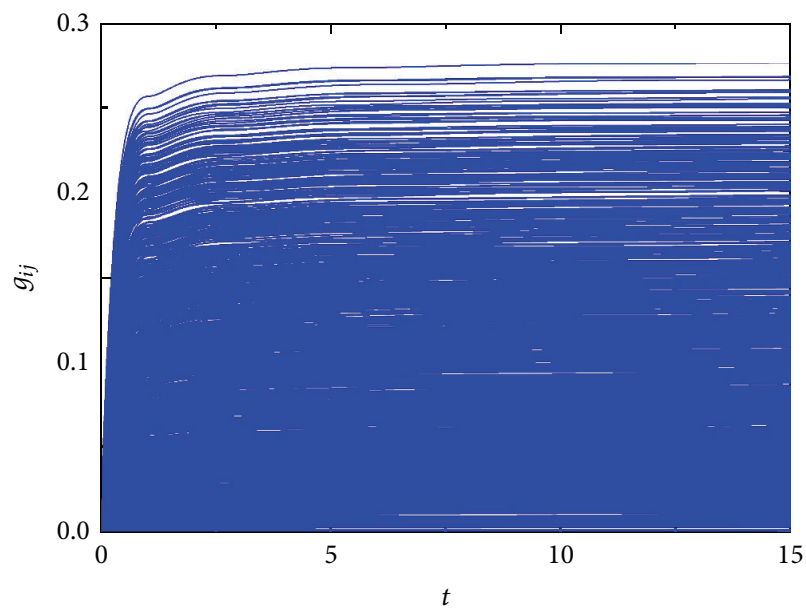

FIGURE 4: Edge strengths' evolution during the interval of the BA scale-free neural network in Figure 2 to realize cluster synchronization.

cluster synchronization with faster cluster synchronization rate, which can be implemented in real networks.

\section{Conclusions}

In this paper, an exponential cluster synchronization control frame of the neural networks with time delay has been established. Different from the prior works, delays in the paper are proportional delays which are unbounded and time-varying, and the coupling strength of each edge in the system only depends on its local information. By constructing the appropriate Lyapunov function and using the inequality 
technique, several sufficient conditions were obtained to make the neural network with the proportional delay realize cluster synchronization. Finally, a numerical simulation has been given to verify the theoretical results. Further the example indicates that the cluster synchronization rate is related to the clusters size. This strategy may be extended to study the effects of the rate of the neural system with proportional delay in order to realize cluster synchronization.

Furthermore we can also extend the current result to the Networked Control Systems (NCS) under scheduling protocol [35-37]. Based on this paper, we can provide a stability certificate that takes into account the network imperfections: communication delays (proportional delays), scheduling protocols, and quantization in order to offer several practical advantages: reduced costs, ease of installation and maintenance, and increased flexibility.

\section{Conflict of Interests}

The authors declare that there is no conflict of interests regarding the publication of this paper.

\section{Acknowledgments}

This work was jointly supported by the National Natural Science Foundation of China (Grants nos. 11375033, 61104152, and 61377067) and the Fundamental Research Funds for the Central Universities (no. 2013XZ02).

\section{References}

[1] X. F. Wang, "Complex networks: topology, dynamics and synchronization," International Journal of Bifurcation and Chaos, vol. 12, no. 5, pp. 885-916, 2002.

[2] L. M. Pecora and T. L. Carroll, "Synchronization in chaotic systems," Physical Review Letters, vol. 64, no. 8, pp. 821-824, 1990.

[3] M. Feki, "An adaptive chaos synchronization scheme applied to secure communication," Chaos, Solitons \& Fractals, vol. 18, no. 1, pp. 141-148, 2003.

[4] A. Pikovsky, M. Rosenblum, and J. Kurths, Synchronization: A Universal Concept in Nonlinear Sciences (Paperback), Cambridge Nonlinear Science Series, 2003.

[5] P. de Lellis, M. di Bernardo, and F. Garofalo, "Synchronization of complex networks through local adaptive coupling," Chaos, vol. 18, no. 3, Article ID 037110, 2008.

[6] W. Ren, "Synchronization of coupled harmonic oscillators with local interaction," Automatica, vol. 44, no. 12, pp. 3195-3200, 2008.

[7] W. Lu, B. liu, and T. Chen, "Cluster synchronization in networks of coupled non-identical dynamical systems," Chaos, vol. 20, no. 1, Article ID 013120, 2010.

[8] Y. Wang and J. Cao, "Cluster synchronization in nonlinearly coupled delayed networks of non-identical dynamic systems," Nonlinear Analysis: Real World Applications, vol. 14, no. 1, pp. 842-851, 2013.

[9] Z. Ma, Z. Liu, and G. Zhang, "A new method to realize cluster synchronization in connected chaotic networks," Chaos, vol. 16, no. 2, Article ID 023103, 2006.
[10] W. Wu, W. Zhou, and T. Chen, "Cluster synchronization of linearly coupled complex networks under pinning control," IEEE Transactions on Circuits and Systems I: Regular Papers, vol. 56, no. 4, pp. 829-839, 2009.

[11] J. Zhou, T. Chen, and L. Xiang, "Robust synchronization of delayed neural networks based on adaptive control and parameters identification," Chaos, Solitons and Fractals, vol. 27, no. 4, pp. 905-913, 2006.

[12] C.-J. Cheng, T.-L. Liao, J.-J. Yan, and C.-C. Hwang, "Exponential synchronization of a class of neural networks with time-varying delays," IEEE Transactions on Systems, Man, and Cybernetics, Part B: Cybernetics, vol. 36, no. 1, pp. 209-215, 2006.

[13] J.-S. Lin, M.-L. Hung, J.-J. Yan, and T.-L. Liao, "Decentralized control for synchronization of delayed neural networks subject to dead-zone nonlinearity," Nonlinear Analysis, Theory, Methods \& Applications, vol. 67, no. 6, pp. 1980-1987, 2007.

[14] X. Li and M. Bohner, "Exponential synchronization of chaotic neural networks with mixed delays and impulsive effects via output coupling with delay feedback," Mathematical and Computer Modelling, vol. 52, no. 5-6, pp. 643-653, 2010.

[15] J. Lu, D. W. C. Ho, J. Cao, and J. Kurths, "Exponential synchronization of linearly coupled neural networks with impulsive disturbances," IEEE Transactions on Neural Networks, vol. 22, no. 2, pp. 329-335, 2011.

[16] X. Yang, Z. Wu, and J. Cao, "Finite-time synchronization of complex networks with non-identical discontinuous nodes," Nonlinear Dynamics, vol. 73, no. 4, pp. 2313-2327, 2013.

[17] W.-T. Miao, T. Yuan, J.-W. Xiao, and Y.-W. Wang, "Adaptive synchronization of complex dynamical networks with two types of time-varying delays," in Proceedings of the 8th IEEE International Conference on Control and Automation (ICCA '10), pp. 1584-1588, IEEE, Xiamen, China, June 2010.

[18] T. Dahms, J. Lehnert, and E. Schöll, "Cluster and group synchronization in delay-coupled networks," Physical Review E, vol. 86, no. 1, part 2, Article ID 016202, 2012.

[19] Y. Chen, M. Hamdi, and D. H. K. Tsang, "Proportional QoS over OBS networks," in Proceedings of the IEEE Global Telecommunicatins Conference (GLOBECOM '01), pp. 1510-1514, November 2001.

[20] J. Wei, C.-Z. Xu, X. Zhou, and Q. Li, "A robust packet scheduling algorithm for proportional delay differentiation services," Computer Communications, vol. 29, no. 18, pp. 3679-3690, 2006.

[21] S. Kulkarni, R. Sharma, and I. Mishra, "New QoS routing algorithm for MPLS networks using delay and bandwidth constrainst," International Journal of Information and Communication Technology Research, vol. 2, no. 3, pp. 285-293, 2012.

[22] J. Liu, D. Yuan, S. Ci, and Y. Zhong, "A new QoS routing optimal algorithm in mobile ad hoc networks based on hopfield neural network," in Advances in Neural Networks-ISNN 2005, vol. 3498 of Lecture Notes in Computer Science, pp. 343-348, Springer, Berlin, Germany, 2005.

[23] R. Gargi, Y. Chaba, and R. B. Patel, "Improving the performance of dynamic source routing protocol by optimization of neural networks," International Journal of Computer Science Issues, vol. 9, Issue 4, no. 3, 2012.

[24] B. van Brunt, J. C. Marshall, and G. C. Wake, "Holomorphic solutions to pantograph type equations with neutral fixed points," Journal of Mathematical Analysis and Applications, vol. 295, no. 2, pp. 557-569, 2004.

[25] Y. Liu, "Asymptotic behaviour of functional-differential equations with proportional time delays," European Journal of Applied Mathematics, vol. 7, no. 1, pp. 11-30, 1996. 
[26] L. Zhou, "Delay-dependent exponential stability of cellular neural networks with multi-proportional delays," Neural Processing Letters, vol. 38, no. 3, pp. 347-359, 2013.

[27] L. Zhou, "Dissipativity of a class of cellular neural networks with proportional delays," Nonlinear Dynamics, vol. 73, no. 3, pp. 1895-1903, 2013.

[28] A. Iserles and Y. Liu, "On neutral functional-differential equations with proportional delays," Journal of Mathematical Analysis and Applications, vol. 207, no. 1, pp. 73-95, 1997.

[29] H. Brunner, Q. Hu, and Q. Lin, "Geometric meshes in collocation methods for Volterra integral equations with proportional delays," IMA Journal of Numerical Analysis, vol. 21, no. 4, pp. 783-798, 2001.

[30] L. Zhou, X. Chen, and Y. Yang, "Asymptotic stability of cellular neural networks with multiple proportional delays," Applied Mathematics and Computation, vol. 229, no. 5, pp. 457-466, 2014.

[31] L. Zhou, "Global asymptotic stability of cellular neural networks with proportional delays," Nonlinear Dynamics, vol. 771, pp. 4147, 2014.

[32] J. Cao and L. Li, "Cluster synchronization in an array of hybrid coupled neural networks with delay," Neural Networks, vol. 22, no. 4, pp. 335-342, 2009.

[33] C.-J. Cheng, T.-L. Liao, J.-J. Yan, and C.-C. Hwang, "Synchronization of neural networks by decentralized feedback control," Physics Letters A: General, Atomic and Solid State Physics, vol. 338, no. 1-18, pp. 28-35, 2005.

[34] A.-L. Barabási and R. Albert, "Emergence of scaling in random networks," Science, vol. 286, no. 5439, pp. 509-512, 1999.

[35] K. Liu, E. Fridmana, and L. Hetel, "Stability and L2-gain analysis of networked control systems under Round-Robin scheduling: a time-delay approach," Systems \& Control Letters, vol. 61, no. 8, pp. 666-675, 2012.

[36] W. P. M. H. Heemels, A. R. Teel, N. van de Wouw, and D. Nešić, "Networked control systems with communication constraints: tradeoffs between transmission intervals, delays and performance," IEEE Transactions on Automatic Control, vol. 55, no. 8, pp. 1781-1796, 2010.

[37] K. Liu, E. Fridman, and L. Hetel, "Network-based control via a novel analysis of hybrid systems with time-varying delays," in Proceedings of the IEEE 51st Conference on Decision and Control (CDC '12), pp. 3886-3891, December 2012. 


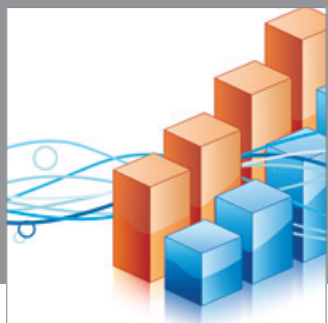

Advances in

Operations Research

mansans

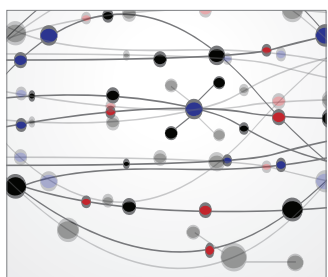

The Scientific World Journal
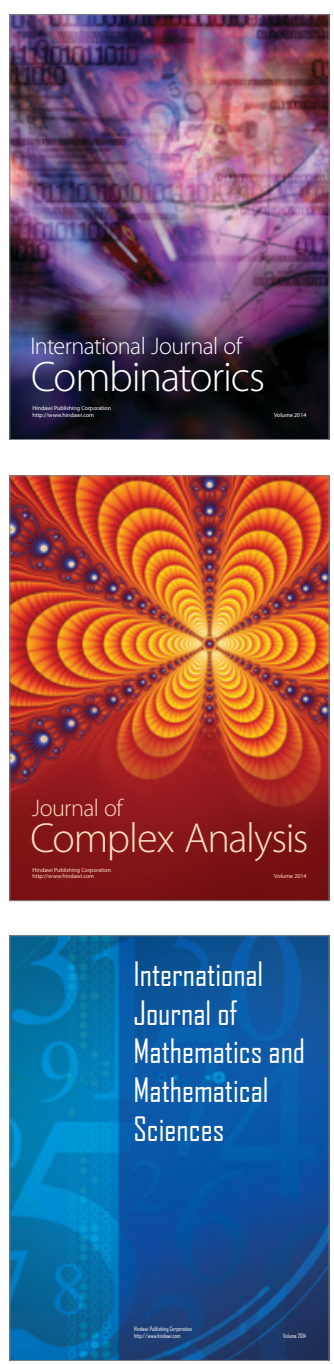
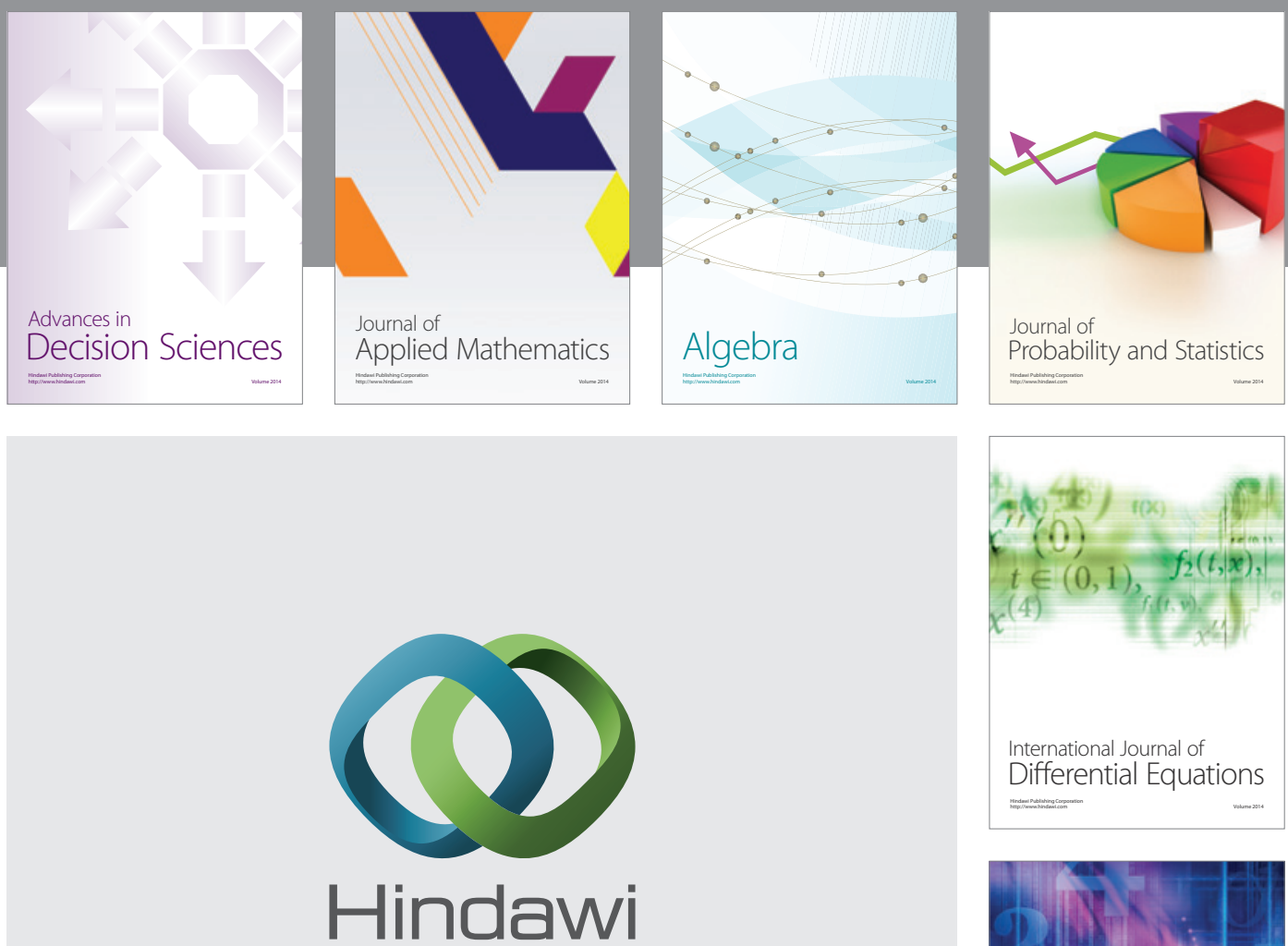

Submit your manuscripts at http://www.hindawi.com
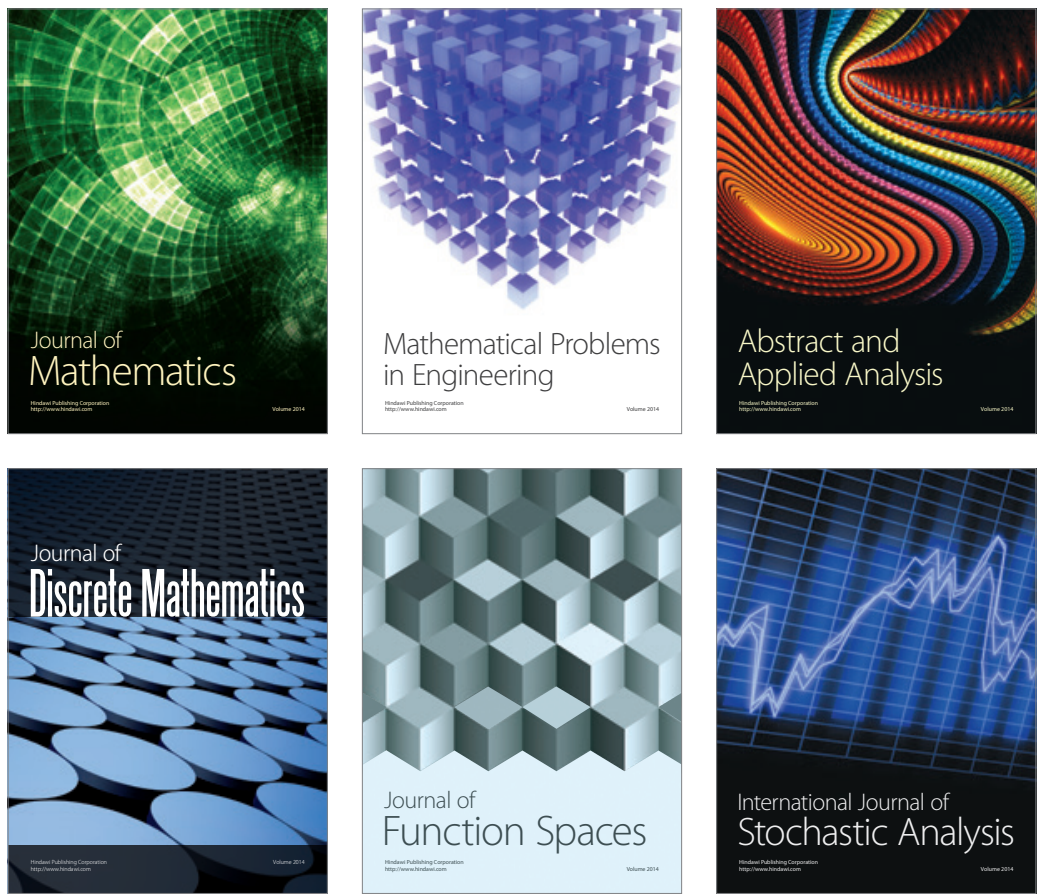

Journal of

Function Spaces

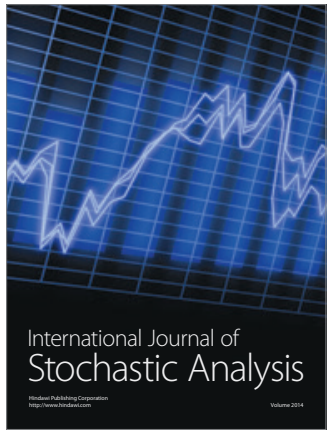


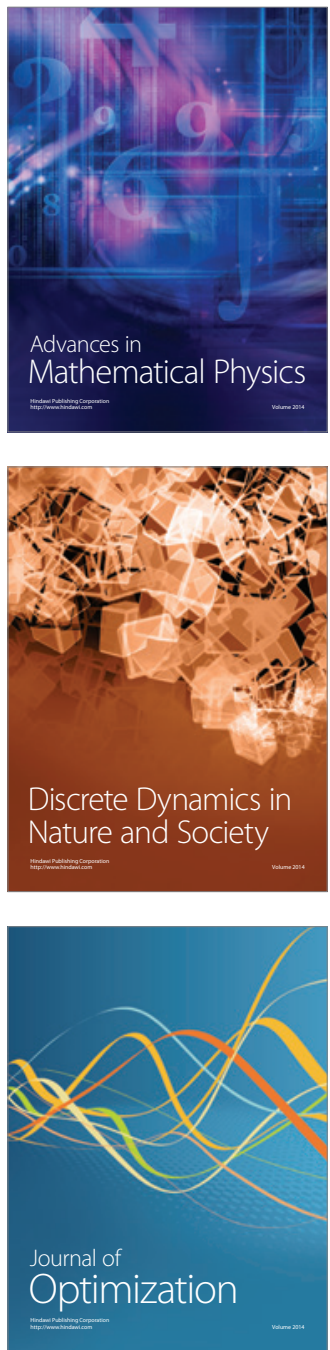